\title{
Prevalence of Cerebrovascular Accidents in Patients with Ulcerative Colitis in a Single Academic Health System
}

\section{Erika Horta}

University of Arkansas for Medical Sciences

Conor Burke-Smith

University of lowa

Bryant Megna ( $\sim$ megna003@umn.edu )

University of Minnesota

Kendall Nichols

University of Minnesota

Byron Vaughn

University of Minnesota

Rwoof Reshi

University of Oklahoma

\section{Eugenia Shmidt}

University of Minnesota

\section{Research Article}

Keywords: stroke, ulcerative colitis, Crohn disease, risk factor

Posted Date: September 30th, 2021

DOI: https://doi.org/10.21203/rs.3.rs-923297/v1

License: (a) (1) This work is licensed under a Creative Commons Attribution 4.0 International License. Read Full License 


\section{Abstract}

Background:

In general, IBD increases arteriovenous thromboembolic events, though the association between UC and cerebrovascular complications remains inconclusive. Some studies suggest young women with UC have an increased risk of cerebrovascular accidents (CVA). The focus of this study was to characterize the rates, anatomic locations and risk factors for CVA in patients with UC.

Methods:

We developed a retrospective cohort of patients with UC at a single health care system from June 2010 to June 2015. Neuroimaging was used to document presence, location and type of stroke and traditional risk factors were considered.

Results:

The prevalence of CVAs increased in both sexes with a peak prevalence of $24.7 \%$ ( $95 \% \mathrm{Cl} 17.1$ - 34.4) in women with UC over the age of 80 . Older age, cancer and atrial fibrillation were risk factors for CVA in univariate analysis for both sexes. In multifactorial analysis, both age and atrial fibrillation were risk factors for CVA in the m-UC cohort, but only age was associated with CVA in f-UC. The most common type of CVA was ischemic stroke (77.7\%). The most common locations for CVAs in UC patients were frontal and occipital lobes ( $19 \%$ and $18 \%$, respectively).

Conclusions:

UC patients have an increased risk for CVA, with women over 80 demonstrating the highest risk. Providers should be aware of these risks in making treatment decisions for UC.

\section{Introduction}

Ulcerative colitis (UC) and Crohn's disease (CD) are the two most common forms of inflammatory bowel disease (IBD) and are thought to arise from an abnormal immune response to the intestinal microbiota in a genetically susceptible host [1]. IBD is considered a prothrombotic state and is associated with an increase in both venous and arterial thromboses [2-4]. This prothrombotic state is driven by a combination of acquired risk factors (e.g. corticosteroids and cigarette smoking), abnormalities of the coagulation cascade (increased fibrinogen and decreased tPA), inflammation, and endothelial dysfunction [5]. Clinical risk for thromboembolism in general is securely linked to IBD, especially in the setting of active disease. However, the drivers of cerebrovascular accidents (CVAs) in these patients remain less well understood.

IBD is associated with chronic inflammation, higher prevalence of atrial fibrillation (AF), hypercoagulability, and various vasculitides; all of which are independently related to the risk of CVA [6, 7]. The literature remains incomplete regarding the universal association between IBD and CVA, with some studies suggesting an increased risk of CVA only in certain subsets of IBD patients. For example, some studies found an increased incidence of CVA in CD, while UC patients were spared [2, 3, 8, 9-11]. On the other hand, specific subgroups of 
UC patients seem to be more affected than others; including younger women (controlling for contraception use), those with concurrent AF, and those with active inflammation $[3,12]$. Moreover, most of the above population-level studies investigating the association between IBD and CVA were conducted outside of the United States (U.S.) which leaves a gap in the literature regarding the distribution of IBD-associated CVAs in this country. Additionally, the typical vascular location of CVAs is not known in IBD patients. The variable CVA rates in subgroups of IBD patients are not at this time fully described or understood.

Our study analyzed primary source data to assess prevalence of CVA in a cohort of patients with UC seen in a large academic and community health care system in Minnesota. We sought to define the nature of CVA in these patients (i.e. vascular localization, ischemic vs hemorrhagic etc.) and to further characterize the associated risk factors.

\section{Methods}

\subsection{Study design}

This study was approved by the Institutional Review Board of the University of Minnesota (IRB\#1501M59201). Informed consent was obtained from all patients included in this study. All methods herein were performed in accordance with relevant guidelines and regulations including the STROBE guidelines for observational studies and the WMA Declaration of Helsinki regarding the ethical principles for medical research involving human subjects. We extracted data from a Clinical Data Repository cultivated via the University of Minnesota/Fairview Health System electronic medical record (EMR). The Clinical Data Repository consists of EMR data from over 2.5 million patients across 8 hospitals and 40 clinics including a large tertiary care academic center and community affiliates. We identified UC patients (ICD 9/10 codes, 556.9/K51.XX) who were evaluated between June 2010 and June 2015, in both the inpatient and outpatient settings.

Demographic and clinical factors were then extracted from the EMR. These included age, sex, race, specific medical comorbidities (AF, cancer) and presence/absence of history of CVA. All patients with radiographic studies of the brain were identified, and these images were reviewed for evidence of CVA, specifically ischemic or hemorrhagic stroke.

To further understand the nature of the increased prevalence in strokes among $\mathrm{f}-\mathrm{UC}$, we randomly matched CD female patients (f-CD) (ICD 9/10 codes, 555.9/K50.XX) by age to $f-U C$ patients seen at the same institution within the same time period. Comparisons between $\mathrm{f}-\mathrm{UC}$ and $\mathrm{f}-\mathrm{CD}$ patients were also analyzed using Pearson's test.

\subsection{Definition of Patients and Conditions}

Diagnoses of UC and CD were established based on typical endoscopic and histologic findings consistent with IBD as interpreted by the treating gastroenterologist or colorectal surgeon. CVA was defined as the presence of hemorrhagic or ischemic stroke on neuroimaging, venous sinus thrombosis, amaurosis fugax, TIA, or history of alteplase administration during a stroke code and/or neurological deficits documented by a neurologist. 


\subsection{Statistical analysis}

The prevalence of CVA in the UC patient cohort was compared to the national and state-level (Minnesota) prevalence of stroke reported by the American Heart Association (AHA) and the Centers for Disease Control and Prevention (CDC) over the same period of time. Patient sex, presence of $A F$, and cancer were treated as binomial variables and Pearson's test was used for this analysis. Kruskal-Wallis test was used when age was treated as a continuous variable. Variables that were statistically significant on univariate analysis were analyzed via multivariate analysis to identify variables that were independently associated with CVA in patients with UC.

All of the above statistical analyses were performed with the use of JMP software (SAS institute, version 15.2.0). When multiple comparisons were done, Bonferroni correction was used and only $p$-values less than 0.006 were considered significant, otherwise, $p$-values $<0.05$ were considered significant.

\section{Results}

A total of 2,183 UC patients were identified. Patient demographics, comorbidities, prevalence and location of CVAs per sex are illustrated in Table 1. 
Table 1

Demographics and comorbidities of patients with IBD

\begin{tabular}{|c|c|c|c|c|c|c|c|c|c|c|c|}
\hline & \multicolumn{3}{|c|}{$f-U C$} & \multicolumn{3}{|c|}{$m-U C$} & Total & p & \multicolumn{3}{|c|}{$f-C D$} \\
\hline & \multicolumn{3}{|c|}{$\mathrm{N}(\%)$} & \multicolumn{3}{|c|}{$N(\%)$} & $N(\%)$ & pearson & \multicolumn{3}{|c|}{$\mathbf{N}(\%)$} \\
\hline $\begin{array}{l}\text { Number of } \\
\text { patients }\end{array}$ & \multicolumn{3}{|c|}{1088} & \multicolumn{3}{|c|}{1095} & 2183 & & \multicolumn{3}{|c|}{1088} \\
\hline Age & \multicolumn{3}{|c|}{$31(2.8)$} & \multicolumn{3}{|c|}{$23(2.1)$} & 54 & 0.45 & \multicolumn{3}{|c|}{$31(2.8)$} \\
\hline$<20$ & \multicolumn{3}{|c|}{$295(27.1)$} & \multicolumn{3}{|c|}{309 (28.2) } & \multirow{2}{*}{$\begin{array}{l}604 \\
(27.7)\end{array}$} & \multirow[t]{2}{*}{1.0} & \multicolumn{3}{|c|}{$295(27.1)$} \\
\hline $20-40$ & \multicolumn{3}{|c|}{388 (35.7) } & \multicolumn{3}{|c|}{369 (33.7) } & & & \multicolumn{3}{|c|}{388 (35.7) } \\
\hline $40-60$ & \multicolumn{3}{|c|}{$281(25.8)$} & \multicolumn{3}{|c|}{$322(29.4)$} & \multicolumn{2}{|l|}{$\begin{array}{l}757 \\
(34.7)\end{array}$} & \multicolumn{3}{|c|}{$281(25.8)$} \\
\hline $60-80$ & \multicolumn{3}{|c|}{$93(8.5)$} & \multicolumn{3}{|c|}{$72(6.6)$} & & & \multirow{2}{*}{\multicolumn{3}{|c|}{$93(8.5)$}} \\
\hline$>80$ & $91 \%$ & & & $90 \%$ & & & (27.6) & & & & \\
\hline & \multicolumn{3}{|l|}{$5 \%$} & \multicolumn{3}{|l|}{$5 \%$} & $\begin{array}{l}167 \\
(7.6)\end{array}$ & & & & \\
\hline \multicolumn{7}{|l|}{ White } & $90 \%$ & & & & \\
\hline \multicolumn{7}{|l|}{ Unknown } & $5 \%$ & & & & \\
\hline & \multicolumn{3}{|c|}{$\begin{array}{l}\text { Stroke } \\
(55)\end{array}$} & \multicolumn{3}{|c|}{ Stroke(47) } & 282 & 0.89 & \multicolumn{3}{|c|}{$\begin{array}{l}\text { Stroke } \\
(33)\end{array}$} \\
\hline Comorbidity & Yes & No & total & Yes & No & Total & & & yes & No & Total \\
\hline Cancer & 13 & 124 & 137 & 17 & 128 & 145 & & & 8 & 128 & 136 \\
\hline & & & $(12.6)$ & & & & & & & & (12.5) \\
\hline Atrial & 10 & 52 & 62 & 19 & 53 & 72 & & & 7 & 55 & 62 \\
\hline & & & (5.7) & & & & $(12.9 \%)$ & 0.58 & & & (5.7) \\
\hline
\end{tabular}

$(6 \%)$

*For those patients that location of stroke was available, patients with multiple stroke locations were counted only once for prevalence, but each location was counted separately for stroke location. CVA: cerebral vascular accident, TIA: transient ischemic attack, SVT: sinus venous thrombosis. BG: Basal Ganglia. 


\begin{tabular}{|c|c|c|c|c|c|}
\hline \multirow{2}{*}{$\begin{array}{l}\text { CVAs* } \\
\text { Hemorrhagic } \\
\text { Ischemic }\end{array}$} & ftet(5.1) & m-l 4.3$)$ & \multirow{2}{*}{$\begin{array}{l}\text { Total } \\
(4.7) \\
N(\%)\end{array}$} & \multirow{2}{*}{$\begin{array}{l}\text { P.4 } \\
\text { pearson }\end{array}$} & \multirow{2}{*}{$\begin{array}{l}\mathrm{B} Q(B .0) \\
\mathrm{N}(\%)\end{array}$} \\
\hline & $\mathrm{N}(\%) 5)$ & $\mathrm{N}(\%) B)$ & & & \\
\hline TIA & $43(78.2)$ & $35(74.5)$ & \multirow{2}{*}{$\begin{array}{l}78 \\
(76.5)\end{array}$} & & $30(91.0)$ \\
\hline SVT & $9(16.4)$ & 9 (19.1) & & & $1(3)$ \\
\hline Location* & 0 & $1(2)$ & \multirow{2}{*}{$\begin{array}{l}18 \\
(17.6)\end{array}$} & & $0(1.0)$ \\
\hline \multirow[t]{6}{*}{ Frontal } & \multirow[t]{6}{*}{$9(20)$} & \multirow[t]{6}{*}{7 (18) } & & & \multirow[t]{6}{*}{$9(20)$} \\
\hline & & & $1(1.0)$ & & \\
\hline & & & $16(19)$ & & \\
\hline & & & $14(16)$ & & \\
\hline & & & $8(9)$ & & \\
\hline & & & $15(18)$ & & \\
\hline Parietal & $11(24)$ & $3(8)$ & $13(15)$ & & $9(20)$ \\
\hline Temporal & $4(9)$ & $4(10)$ & $12(14)$ & & $6(13)$ \\
\hline Occipital & $5(11)$ & $10(25)$ & $7(8)$ & & $6(13)$ \\
\hline BG & $6(13)$ & $7(18)$ & & & $7(15)$ \\
\hline Cerebellum & $7(16)$ & $5(13)$ & & & $8(17)$ \\
\hline Brainstem & $3(7)$ & $4(10)$ & & & $1(2)$ \\
\hline
\end{tabular}

Cerebral imaging was obtained in $19.9 \%$ of UC patients. The prevalence of CVA in UC patients was $4.7 \%$, which was higher than the reported prevalence in the U.S. (per AHA and CDC) and in Minnesota. (figure 1a). Prevalence of stroke in m-UC was $4.3 \%,(95 \% \mathrm{Cl} 3.2$ - 5.7), which is higher than the CDC data for stroke in men, at $2.7 \%(95 \% \mathrm{Cl} 2.6-2.8, \mathrm{p}=0.0012)$. Prevalence of stroke in $\mathrm{f}-\mathrm{UC}$ was $5.1 \%,(95 \% \mathrm{Cl} 3.9-6.5)$, which is greater than the $\mathrm{CDC}$ data for stroke in women $(2.6 \% \mathrm{Cl} 2.5-2.7, \mathrm{p}<0.0001)$. Comparing the prevalence of CVAs per age and sex to the AHA data (figure 1b), f-UC over 80 years old were at disproportionately higher risk for CVA with a prevalence of $24.7 \%(p=0.0029)$. Moreover, $m-U C$ between $20-40$ years old had a higher prevalence of CVA than the US population according to AHA data $(p=0.0024)$, but the event rate of CVA was rare with a prevalence of less than $1 \%$. IBD subclass comparison illustrated that the prevalence of stroke in $\mathrm{f}-\mathrm{CD}$ was $3.0 \%$ (vs $5.1 \%$ in $f-U C, p=0.02$ ), and that it increased with age, though was consistently lower than f-UC across all age groups (Figure 1b).

Known thrombogenic risk factors for ischemic CVA, specifically cancer and AF, were investigated. The prevalence of both comorbidities was similar between f-uc and m-uc (Table 1). On univariate analysis, cancer, AF and age were statistically significant risk factors for strokes in $\mathrm{f}-\mathrm{UC}$ and $\mathrm{m}$-UC (Table 2). On multivariate analysis, only AF and age were risk factors for CVA among all UC patients. On multivariate analysis only age 
was a risk factor in f-UC while age, and AF were independently associated risk factors in m-uc. (Table 2) CVA subtype is further delineated in Table 1 . The anatomic localizations of strokes were not different between $\mathrm{f}$-UC and $\mathrm{m}-\mathrm{UC}$

Table 2

Univariate and multivariate analyses for associations between CVAs and age, atrial fibrillation and cancer per group

\begin{tabular}{|c|c|c|c|c|c|c|}
\hline & \multicolumn{3}{|l|}{ Univariate analysis } & \multicolumn{3}{|c|}{ Multivariate analysis } \\
\hline & age & $\mathrm{AF}$ & Cancer & age & $\mathrm{AF}$ & Cancer \\
\hline \multirow[t]{2}{*}{ UC } & $p<0.0001$ & $p<0.0001$ & $p<0.0001$ & $p<0.0001$ & $p=0.020$ & $p=0.66$ \\
\hline & OR 0.0006-0.926 & OR 4.7-12 & OR 1.9-4.7 & OR 10-89* & OR 1.4-4.0 & \\
\hline \multirow[t]{4}{*}{$f-U C$} & $\mathrm{p} 0<.0001$ & $p<0.0001$ & $p=0.011$ & $p<0.0001$ & $p=0.99$ & $p=0.48$ \\
\hline & OR 0.0005-0.925 & OR 2.0-8.8 & OR 1.2-4.3 & OR 13.8-833* & & \\
\hline & & & & OR 7.6-50** & & \\
\hline & & & & OR $1.9-7.4^{\star \star \star}$ & & \\
\hline \multirow[t]{5}{*}{ m-UC } & $p<0.0001$ & $p<0.0001$ & $p<0.0001$ & $p=0.0009$ & $p<0.0001$ & $p=0.11$ \\
\hline & OR 0.0010-0.928 & OR 6.7-24 & OR 2.2-7.6 & OR $0.06-0.7^{\wedge}$ & OR 2.5-10 & \\
\hline & & & & OR $0.09-0.7^{\wedge \wedge}$ & & \\
\hline & & & & OR 0.03-0.4* & & \\
\hline & & & & OR $0.04-0.4^{\star *}$ & & \\
\hline \multirow[t]{5}{*}{$f-C D$} & $p<0.0001$ & $p<0.0001$ & $p=0.04$ & $p=0.0004$ & $p=0.72$ & $p=0.11$ \\
\hline & OR 0.005-0.948 & OR 2.0-12 & OR 1.0-5.2 & OR $0.008-0.47^{\wedge}$ & & \\
\hline & & & & OR $0.1-0.8^{\wedge \wedge}$ & & \\
\hline & & & & OR $0.006-0.4^{*}$ & & \\
\hline & & & & OR $0.08-0.8^{* *}$ & & \\
\hline \multicolumn{7}{|c|}{$\begin{array}{l}\text { *Comparing } 20-40 \text { years old to over } 80 \text { years old. ** Comparing } 40-60 \text { years old to over } 80 \text { years old *** } \\
\text { Comparing } 60-80 \text { years old to over } 80 \text { years old. ^ Comparing } 20-40 \text { years old to } 60-80 \text { years old } \wedge \wedge \\
\text { Comparing } 40-60 \text { years old to 60-80 years old, AF: atrial fibrillation, UC: ulcerative colitis, f-UC: females } \\
\text { with UC, m-UC: males with UC, f-CD: females with Crohn disease. }\end{array}$} \\
\hline
\end{tabular}

\section{Discussion}

In this large, single health system, retrospective chart review, we found that CVA prevalence was higher in our cohort of UC patients than in Minnesota and the U.S. at large. The prevalence of CVA in all UC patients increased with age and the most common stroke subtype was ischemic (70\%). The predominantly ischemic nature of infarction in our dataset agrees with most studies. However, the majority of stroke literature in IBD patients has presented CVAs as an issue of younger, otherwise healthy patients with severe IBD $[2,3]$. In 
contrast, our study found traditional risk factors for CVA to be common in UC. The fact that our study captured a significant degree of community (outside of tertiary center) UC cases may account for this difference.

Patterns of stroke localization are important to understand underlying pathophysiology. A relatively recent review article demonstrated that the majority of strokes identified in IBD patients were in the left or right middle cerebral artery, making anterior circulation events the most commonly affected area, similar to the general population $[9,13]$. A small series of patients with CD identified recurrent posterior circulation strokes, but the three cases described were derived from patients with recurrent strokes, which may have led to biases in the selection of patients with a predilection for this area[14]. In future studies, cerebral imaging with vascular reconstruction should be included for all patients with IBD experiencing CVAs, such to better inform this issue.

An important finding in our study is that post-menopausal women with UC had the highest risk for stroke. This finding contrasts with previous work that showed younger women with UC at greatest risk [3, 12]. Specifically, $\mathrm{Ha}$ et al. compared women with IBD (pooled UC and CD) under age 40 to age-matched healthy controls, and found young women with IBD to have a greater risk of stroke [3]. Variations in hormone levels and/or varying degrees of other cumulative sex-specific risk factors, such as hormone contraception use, may be important contributing factors to this finding. Other investigators have demonstrated that women with IBD are at increased risk for cardiovascular disease and it is presumed that hormonal variations may impart a degree of this risk $[7,11,15]$. Possible explanations are hormonal disequilibrium, inflammation, and endothelial dysfunction [16]. In this way, estrogen has myriad effects on cardiovascular health as women age and this dynamic may persist and possibly be exacerbated in the setting of IBD [17]. This is evidenced by literature supporting the role of estrogen in TNF-alpha modulation as it relates to inflammation and interactions within the gut microbiome [18]. Further, murine studies have shown that estradiol downregulates TNF-alpha and subsequently is protective against acute colitis [19]. These findings represent an intriguing avenue of investigation to further link post-menopausal women with IBD, variations in hormone levels, and increased cerebrovascular risk.

Limitations of our study include both its retrospective nature and lack of correlative data to indicate degree of disease activity in the IBD patients at the time of CVA. Further, more generally, our data did not capture personal history of thrombophilia, tobacco smoking history, immobilization, recent surgery, use of central venous catheters, VTE prophylactic measures, IBD medications, and overall compliance. Missing data and differential loss to follow up may be products of our retrospective design. Our strict definition of stroke prioritized specificity over sensitivity and could have resulted in a lower stroke detection rate in our UC and female CD cohorts. However, an artificially low stroke detection rate should have favored the hypothesis that thromboembolism would not be increased in both cohorts, which suggests that our findings are not spurious. Further, IBD disease activity as illustrated via serum C-reactive protein (CRP) levels, fecal calprotectin levels or better yet, endoscopic impression would allow inferences to be made concerning degree of inflammation and thrombotic risk. This is important, as while quiescent disease exhibits a measurable degree of risk, active IBD flares generate the highest risk of VTE propagation, atrial fibrillation, and stroke [10, 20]. Further, increased disease activity is also linked to higher risk of myocardial infarction, stroke, and cardiovascular-related death, with elevated CRP levels acting as a surrogate for disease activity [21]. Lastly, while it is important that we 
incorporated active cancer and atrial fibrillation, a more comprehensive co-variate model including additional known risk factors for CVA may have yielded a more comprehensive analysis.

\section{Conclusions}

In conclusion, we have demonstrated that, compared to state and nationwide general populations, females with ulcerative colitis are at an increased risk for stroke, especially as they age beyond 80 . Therefore, mitigation of risk factors for ischemic stroke, including aspirin prescription as stroke prevention, should be addressed by gastroenterologists, cardiologists and neurologists taking care of patients with UC. In our study, although cancer and atrial fibrillation were prevalent in UC patients experiencing strokes, only older age persisted as a risk factor for strokes in women with UC. Also, atrial fibrillation may exist as a contributor to strokes in men with ulcerative colitis. Further studies are needed to determine the distinct etiologies for the increased prevalence of CVA in patients with all subtypes of IBD and to further delineate sex-related risk. Ideally, these future investigations will help to design individualized primary and secondary stroke prevention strategies to improve cardiovascular outcomes in patients with IBD.

\section{Declarations}

\section{Disclosures:}

Dr. Vaughn has received personal compensation for speaking/consulting from Abbvie as well as grant support from Genentech, Takeda, Celgene, and Diasorin. Dr. Shmidt has received research grant support from Takeda, Celgene and UCB. All other authors have no disclosures, financial or otherwise.

\section{Author Conflict of Interest/Study Support:}

- Guarantor: Eugenia Shmidt, MD

- Corresponding author: Bryant W. Megna, MD

- Specific Author Contributions:

1. EH: primary drafting, data gathering, statistics, inception, design, literature review

2. CB: data gathering and organization, draft review

3. BWM: drafting, draft review, literature review, data organization

4. KJN: data gathering and organization

5. BPV: draft review, design, methodology

6. RR: draft review, design, methodology

7. ES: draft review, final draft approval, methodology, design, article guarantor

- Financial Support: None

- Potential Competing Interests: None. See above "Disclosures" section for general information on author disclosures.

\section{Declarations:}




\section{Investigation Guidelines:}

Our work adhered to the STROBE guidelines for observational studies. See Addendum 1. Below.

\section{References}

1. Abraham, C. \& Cho, J. H. Inflammatory bowel disease. N Engl J Med, 361, 2066-2078 (2009).

2. Bernstein, C. N., Wajda, A. \& Blanchard, J. F. The incidence of arterial thromboembolic diseases in inflammatory bowel disease: a population-based study. Clin Gastroenterol Hepatol, 6, 41-45 (2008).

3. Ha, C., Magowan, S., Accortt, N. A., Chen, J. \& Stone, C. D. Risk of arterial thrombotic events in inflammatory bowel disease. Am J Gastroenterol, 104, 1445-1451 (2009).

4. Scoville, E. A. et al. Venous thromboembolism in patients with inflammatory bowel diseases: a casecontrol study of risk factors. Inflamm Bowel Dis, 20, 631-636 (2014).

5. Giannotta, M., Tapete, G., Emmi, G., Silvestri, E. \& Milla, M. Thrombosis in inflammatory bowel diseases: what's the link? Thromb J, 13, 14 (2015).

6. Jiang, J. X., Keat, K. \& Swaminathan, S. ANCA-Associated Vasculitis in Inflammatory Bowel Disease. Dig Dis Sci, 64, 3350-3354 (2019).

7. Kristensen, S. L. et al. O. Increased risk of atrial fibrillation and stroke during active stages of inflammatory bowel disease: a nationwide study Europace 2014;16:477-484; Singh S, Kullo IJ, Pardi DS, Loftus EV, Jr. Epidemiology, risk factors and management of cardiovascular diseases in IBD Nat Rev Gastroenterol Hepatol. 2015;12:26-35.

8. Andersohn, F., Waring, M. \& Garbe, E. Risk of ischemic stroke in patients with Crohn's disease: a population-based nested case-control study Inflamm Bowel Dis. 2010;16:1387-1392; L. R. CB, J. Uncommon causes of stroke. City: Cambridge University Press; 2008.

9. Katsanos, A. H. et al. S. Cerebral arterial infarction in inflammatory bowel diseases Eur J Intern Med 2014;25:37-44.

10. Zoller, B., Li, X., Sundquist, J. \& Sundquist, K. Autoimmune diseases and venous thromboembolism: a review of the literature. Am J Cardiovasc Dis, 2, 171-183 (2012).

11. Singh, S., Singh, H., Loftus, E. V. Jr. \& Pardi, D. S. Risk of cerebrovascular accidents and ischemic heart disease in patients with inflammatory bowel disease: a systematic review and meta-analysis. Clin Gastroenterol Hepatol, 12, 382-393 e381: quiz e322. (2014).

12. Kristensen, S. L. et al. J. Disease activity in inflammatory bowel disease is associated with increased risk of myocardial infarction, stroke and cardiovascular death-a Danish nationwide cohort study PLoS One 2013;8:e56944.

13. Chung, J. W. et al. Kim N. Trial of ORG 10172 in Acute Stroke Treatment (TOAST) classification and vascular territory of ischemic stroke lesions diagnosed by diffusion-weighted imaging J Am Heart Assoc 2014;3; Carrera E, Maeder-Ingvar M, Rossetti AO, Devuyst G, Bogousslavsky J, Lausanne Stroke R. Trends in risk factors, patterns and causes in hospitalized strokes over 25 years: The Lausanne Stroke Registry Cerebrovasc Dis. 2007;24:97-103. 
14. Shaban, A., Hymel, B., Chavez-Keatts, M., Karlitz, J. J. \& Martin-Schild, S. Recurrent posterior strokes in inflammatory bowel disease patients. Gastroenterol Res Pract, 2015, 672460 (2015).

15. Babickova, J. et al. Lengyelova E. Sex Differences in Experimentally Induced Colitis in Mice: a Role for Estrogens Inflammation 2015;38:1996-2006.

16. Newson, L. Menopause and cardiovascular disease. Post Reprod Health, 24, 44-49 (2018).

17. Novella, S., Heras, M., Hermenegildo, C. \& Dantas, A. P. Effects of estrogen on vascular inflammation: a matter of timing. Arterioscler Thromb Vasc Biol, 32, 2035-2042 (2012).

18. Baker, J. M., Al-Nakkash, L. \& Herbst-Kralovetz, M. M. Estrogen-gut microbiome axis: Physiological and clinical implications. Maturitas, 103, 45-53 (2017).

19. Armstrong, C. M., Allred, K. F., Weeks, B. R., Chapkin, R. S. \& Allred, C. D. Estradiol Has Differential Effects on Acute Colonic Inflammation in the Presence and Absence of Estrogen Receptor beta Expression. Dig Dis Sci, 62, 1977-1984 (2017).

20. Grainge, M. J., West, J. \& Card, T. R. Venous thromboembolism during active disease and remission in inflammatory bowel disease: a cohort study., 375, 657-663 (2010).

21. Dregan, A., Charlton, J., Chowienczyk, P. \& Gulliford, M. C. Chronic inflammatory disorders and risk of type 2 diabetes mellitus, coronary heart disease, and stroke: a population-based cohort study., 130, 837-844 (2014).

\section{Figures}

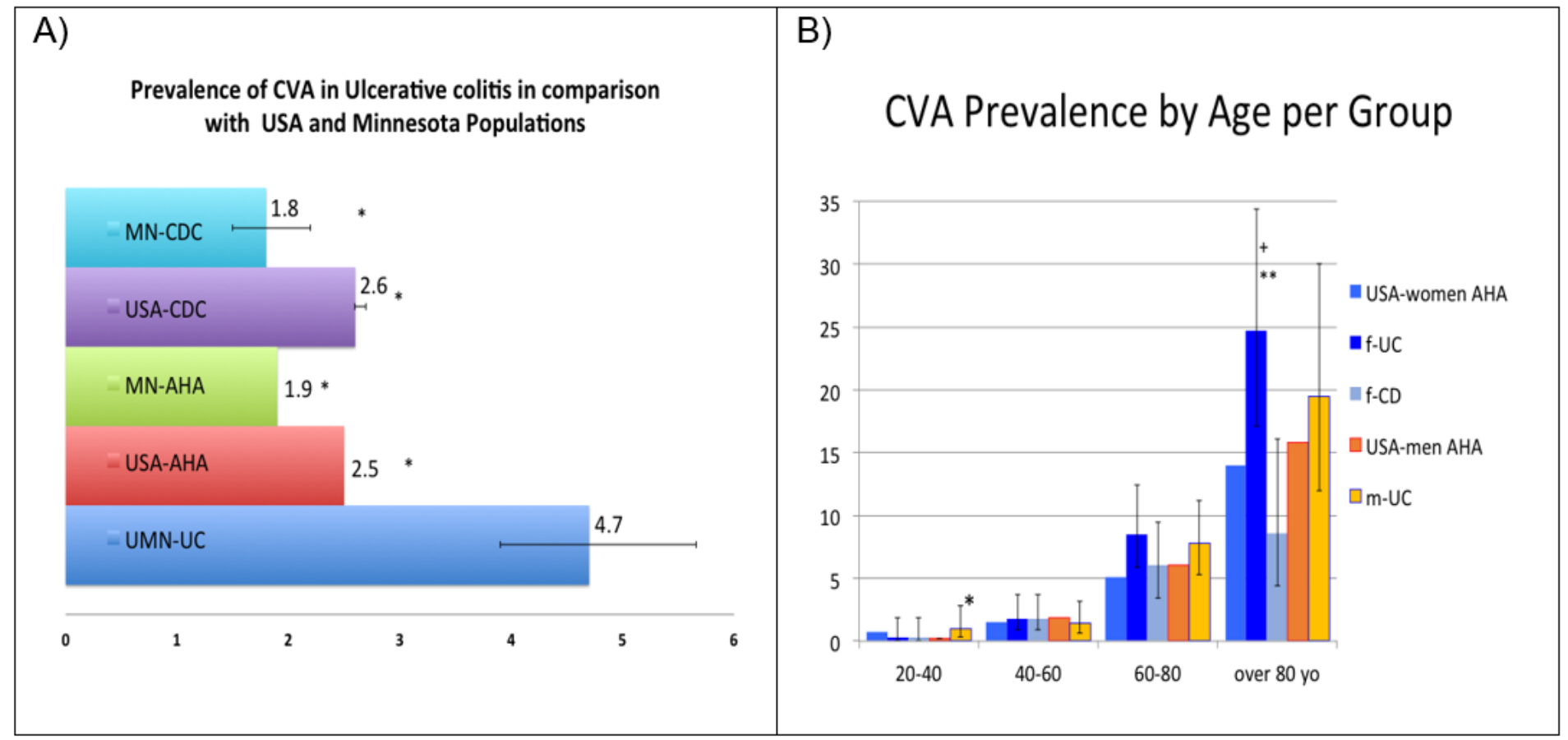

\section{Figure 1}

a) prevalence of cerebral vascular accidents in ulcerative colitis patients in comparison with country and state data, * $p<0.0001$ b)CVA prevalence by age and group, ${ }^{*} p=0.0024$ when comparing with USA-men AHA, ** 
$p=0.0029$ when comparing with USA-women $\mathrm{AHA},+\mathrm{p}=0.0003$ when comparing with UMN-women CD; CVA: cerebrovascular accident, MN: Minnesota, CDC: Centers for Disease Control and Prevention, AHA: American Heart Association, UC: ulcerative colitis, yo: years old 